\title{
Gestion du parc agroforestier du terroir de Vipalogo (Burkina Faso) : contribution des ligneux à la satisfaction des besoins des populations
}

\author{
Georges YAMEOGO ${ }^{1}$, Barthélémy YELEMOU ${ }^{2 *}$, Issiaka Joseph BOUSSIM ${ }^{3}$ et \\ Dossohoua TRAORE $^{4}$ \\ ${ }^{1}$ Institut de l'Environnement et de Recherches Agricoles, 04 BP 8645 Ouagadougou 04, Burkina Faso. \\ ${ }^{2}$ Institut de l'Environnement et de Recherches Agricoles, BP 10 Saria, Burkina Faso. \\ ${ }^{3}$ UFR Sciences de la Vie et de la Terre, Université de Ouagadougou 03 BP 7021 Ouagadougou 03, \\ Burkina Faso. \\ ${ }^{4}$ UFR Biosciences, Université de Cocody, 22 BP 582 Abidjan 22, RCI. \\ *Auteur correspondant, E-mail : yelbart@hotmail.com
}

RESUME

Au Burkina Faso, l'arbre rural fait partie des systèmes de production. Le choix des espèces, leur densité, les modes de gestion ainsi que les besoins à satisfaire, obéissent à des critères propres aux producteurs. C'est pour comprendre ces logiques paysannes de la gestion du parc agroforestier que la présente étude a été initiée dans le terroir de Vipalogo. 66 exploitations, soit 14\% du nombre total des exploitations du terroir ont été enquêtées. Les résultats montrent que la plantation, la régénération naturelle assistée, la coupe (totale ou partielle) sont les principaux modes de gestion du parc. L'utilisation de l'un ou l'autre mode de gestion est fonction du statut foncier. Les arbres du parc sont principalement utilisés pour satisfaire les besoins alimentaires et médicinaux (humains et animaux). Alors que les besoins en produits forestiers non ligneux sont satisfaits en grande partie par les arbres du parc, les produits ligneux sont récoltés principalement dans les zones non cultivées. Au regard du rôle joué par le parc agroforestier et compte tenu du faible taux de régénération des espèces qui le composent, il apparaît nécessaire de promouvoir des modes de gestion efficaces afin d'insuffler un meilleur dynamisme au parc agroforestier.

(C) 2013 International Formulae Group. All rights reserved.

Mots clés: Parc agroforestier, mode de gestion, satisfaction des besoins, Vipalogo, Burkina Faso.

\section{INTRODUCTION}

Depuis trois décennies, le Sahel est confronté à de nombreuses difficultés parmi lesquelles la dégradation du couvert végétal. Cette dégradation a entre autre pour conséquences l'amenuisement des ressources ligneuses et la dégradation des sols, ce qui suscite de nos jours beaucoup d'inquiétudes.

Avec la régression progressive des formations végétales naturelles au profit des terres de culture, une place importante devra être accordée à la gestion de l'arbre au sein de l'exploitation agricole. En effet, avec la saturation foncière, il y a lieu de voir dans quelle mesure l'exploitation agricole peut contribuer à la satisfaction des besoins non seulement en produits agricoles mais aussi en produits ligneux et non ligneux. Cela est d'autant plus important qu'en milieu rural, les femmes parcourent de longues distances pour s'approvisionner en bois de chauffe et également en produits forestiers non ligneux qui interviennent pour beaucoup dans la satisfaction des besoins alimentaires. Pour Thiombiano et al. (2012), l'arbre joue un rôle important dans les stratégies de survie des 
populations rurales frappées par de longues années de sécheresse, en raison non seulement de la richesse nutritive de certains produits non ligneux issus des arbres et arbustes de la zone tropicale.

La nécessité d'asseoir des règles de gestion durable des ressources ligneuses a été abordée notamment au Nord-Cameroun, dans la région de MAFA-KILDA, au regard de l'état de dégradation du milieu (ENGREF, 2001). Pour connaître les pratiques de gestion des ressources arborées par les femmes, Gautier et al. (2001) ont mené des études dans cinq villages du Nord-Cameroun.

$\mathrm{Au}$ Burkina Faso, pays sahélien à écosystèmes fragiles, les paysages agraires sont caractérisés par l'omniprésence d'une strate arborée dans les cultures, donnant ainsi naissance aux parcs agroforestiers. Ces parcs sont constitués d'espèces qui fournissent des produits surtout alimentaires, répondant ainsi aux besoins des sociétés qui les élaborent (Yameogo et al., 2005).

Outre la satisfaction des besoins domestiques, les arbres du parc contribuent à la réduction de l'évapotranspiration, fournissent un ombrage qui tempère l'intensité $\mathrm{du}$ soleil pour les hommes, les animaux et certaines plantes cultivées ou spontanées (Millogo-Rasolodimby, 2001).

Du point de vue biologique, les arbres assurent la remontée, le stockage et la restitution d'éléments minéraux. Les ligneux peuvent capter sur leur feuillage des dépôts éoliens fins et des excréments d'oiseaux qui contribuent à enrichir le sol sous leur houppier (Yélémou, 2010). Certaines espèces comme Faidherbia albida Del., Piliostigma reticulatum (DC) Hochst., peuvent améliorer la fertilité du sol et contribuer à augmenter les rendements du mil, du sorgho et du maïs (Traoré et al., 2007 ; Yélémou, 2010).

Dans le parc agroforestier, le choix des espèces, leur densité, les besoins à satisfaire, de même que les modes de gestion des arbres, obéissent à des règles propres aux producteurs. Comprendre la gestion des ressources naturelles, notamment agroforestières, apparaît donc comme une problématique de premier plan qui permettra d'améliorer durablement la productivité des écosystèmes cultivés. Pour Thiombiano (2005), la diversité des ressources biologiques constitue depuis quelques décennies un thème central autour duquel plusieurs disciplines conjuguent leurs efforts non seulement pour sa sauvegarde mais aussi pour son utilisation durable. C'est pour comprendre le système actuel de gestion des arbres dans le parc agroforestier en vue d'une meilleure gestion, que la présente étude a été initiée dans le terroir de Vipalogo. Elle devra permettre, (i) la détermination des pratiques sylvicoles appliquées aux arbres se trouvant dans les champs, (ii) l'identification de la contribution des arbres du parc à la satisfaction des besoins des exploitations agricoles.

\section{MATERIEL ET METHODES}

\section{Localisation et caractérisation du site}

Le site de l'étude est Vipalogo, un village situé à $40 \mathrm{~km}$ au Sud Ouest de Ouagadougou (capitale du Burkina Faso et chef-lieu de la province du Kadiogo). Il est compris entre la latitude $12^{\circ} 04^{\prime} 20^{\prime}$ ' et $12^{\circ} 09^{\prime} 30^{\prime}$ ' nord et $1^{\circ} 42^{\prime} 50^{\prime}$ ' et $1^{\circ} 49^{\prime} 00^{\prime}$ ', ouest (Figure 1).

Le terroir de Vipalogo fait partie du grand ensemble généralement appelé «Plateau Central» qui se caractérise généralement par une forte concentration humaine de 76 habitants au $\mathrm{km}^{2}$, contre une moyenne nationale de 38 habitants au $\mathrm{km}^{2}$ (MEDEV/DGEP, 2005). On note aussi une intense occupation qui entraîne la dégradation des terres et des conflits assez fréquents entre agriculteurs et entre agriculteurs et éleveurs.

Vipalogo appartient au domaine phytogéographique Nord-Soudanien (Fontes \& Guinko, 1995) caractérisé par des savanes arborées et arbustives à Vitellaria paradoxa Gaertn.f., Lannea microcarpa Engl. \& K. Krause, Parkia biglobosa (Jacq.) R.Br.ex G.Don., Faidherbia albida (Del.) Chev., Tamarindus indica L., Combretum micranthum G. Don, Combretum glutinosum Perr. ex DC. , Guiera senegalensis J.F.Gmel.; Acacia seyal Del.. Le paysage est fortement marqué par l'emprise humaine, illustrée par la présence de savanes parcs et de jachères à karité (Vitellaria paradoxa) et à néré (Parkia biglobosa).

Le climat est caractérisé par une saison sèche de sept mois (de novembre à mai) et une saison pluvieuse de cinq mois (de juin à octobre). La pluviométrie annuelle est très 
variable et oscille entre $600 \mathrm{~mm}$ et $1000 \mathrm{~mm}$, avec une moyenne de $761 \mathrm{~mm}$ sur les trente dernières années. Les températures moyennes mensuelles connaissent également une variation allant de $24^{\circ} \mathrm{C}$ à $33^{\circ} \mathrm{C}$.

\section{Méthodes}

\section{Echantillonnage}

L'unité d'observation est l'exploitation agricole ou ménage retenu par échantillonnage aléatoire. Le premier degré d'échantillonnage est constitué des quartiers du village au nombre de cinq (05) et le second degré, des exploitations agricoles au nombre de 409. Au sein de chaque quartier, six (06) exploitations ont été retenues et 36 au niveau du second degré, de manière aléatoire et simple. Au total, 66 exploitations ont été enquêtées, soit $14 \%$ des exploitations du terroir.

\section{Dispositif et outils de collecte des informations}

Les enquêtes ont été menées en discussion de groupe et en interviews individuelles auprès des ménages du terroir de
Vipalogo. Les outils utilisés ont été les entretiens semi structurés s'articulant autour des quatre points suivants :

- le statut foncier des champs et des jachères ainsi que leur localisation ;

- les pratiques agroforestières ;

- la gestion des arbres dans les champs ;

- les besoins satisfaits par les arbres.

\section{Analyse des données}

Les données collectées sont de nature qualitative. Elles ont donc été codifiées et traitées à l'aide des logiciels Excel et SPSS pour faire ressortir les modes de gestion en fonction de différents paramètres dont le statut foncier, le type de champ, les besoins à satisfaire. Au niveau des types de champs, nous avons distingué les champs de case qui sont les champs autour des concessions villageoises et les champs de brousse qui sont ceux éloignés du village.

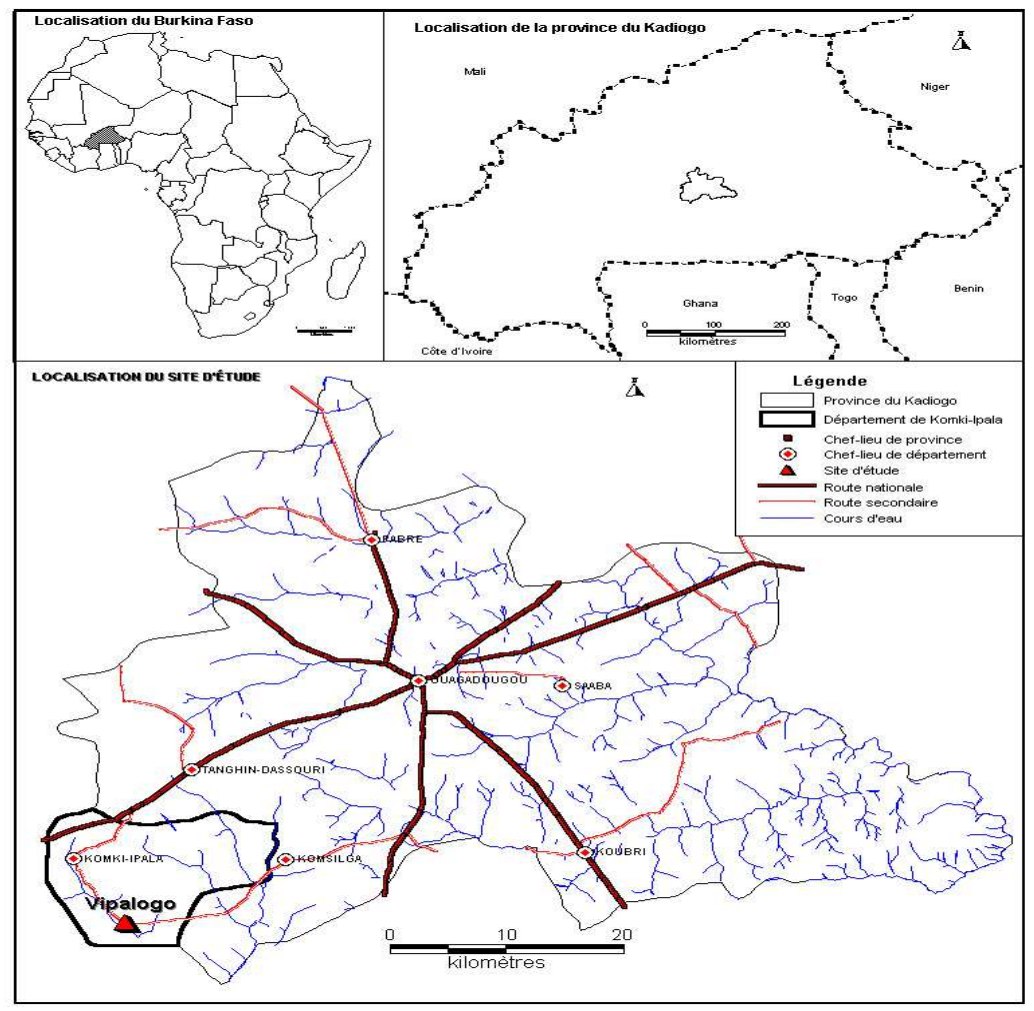

Figure 1: Localisation du site de Vipalogo. 


\section{RESULTATS}

L'enquête a permis de déterminer que les modes de gestion appliqués aux arbres des parcs agroforestiers du terroir de Vipalogo étaient la coupe (totale ou partielle), la régénération naturelle assistée (RNA) et la plantation.

\section{Les techniques de coupes appliquées}

Un tiers des personnes questionnées disent pratiquer la coupe des arbres. Cette pratique varie cependant en fonction des types de champs. $45,5 \%$ disent couper exclusivement des arbres dans leurs champs de case, tandis que seulement $18,2 \%$ ne coupent que dans leurs champs de brousse. $36,3 \%$ exploitent les arbres aussi bien dans leurs champs de case que dans leurs champs de brousse.

\section{Trois pratiques différentes sont utilisées La coupe complète au ras du sol}

Elle consiste à abattre l'arbre. Il faut distinguer la coupe de défrichement, qui s'effectue généralement lors du défrichement de nouveaux champs, et la coupe «sylvicole», qui s'effectue dans les parcs arborés et les plantations. Dans les champs déjà cultivés, l'abattage concerne essentiellement les espèces exotiques, telles que le neem Azadirachta indica A.Juss. et Eucalyptus camaldulensis Dehnh., dans le but de satisfaire les besoins en bois de service et d'énergie. Les espèces locales du parc sont rarement abattues, à l'exception de vieux pieds non productifs ou ayant des anomalies très prononcées tels de grands trous à la base. Cela participe également de la gestion du parc par la conservation des arbres les plus productifs et les plus vigoureux.

\section{La coupe partielle ou émondage partiel}

C'est une technique connue par les producteurs de Vipalogo (Photo 1). 98\% des enquêtés disent connaître la technique qui consiste à tailler les branches à une certaine hauteur pour favoriser l'accès aux fruits ou aux feuilles. Dans les faits, elle est pratiquée par 30,3\% de l'échantillon. Elle concerne la plupart du temps des espèces locales telles que le néré (Parkia biglobosa), le tamarinier (Tamarindus indica) et le kapokier (Bombax costatum Pellegr. et Vuillet.).

\section{L'étêtage}

C'est une pratique qui consiste à couper la cime de l'arbre. Les Photos 2 et 3 présentent respectivement un pied de neem et de néré étêtés.

L'étêtage est connu à Vipalogo et appliqué par plusieurs producteurs, essentiellement sur des vieux pieds ou sur des arbres fortement parasités. Les principales espèces agroforestières qui sont concernées par cette pratique sont, par ordre de fréquence: Parkia biglobosa, Vitellaria paradoxa, Azadirachta indica, Bombax costatum, Ficus capensis Thunb., Lannea microcarpa, Mangifera indica L., Psidium guajava L., Cassia sieberiana DC. et Tamarindus indica.

Deux tiers des cultivateurs, soit 44 chefs d'exploitation, utilisent cette pratique qui est influencée par la localisation des champs (Tableau 1). Comme le montre ce tableau, l'étêtage est plus utilisé dans les champs de brousse que dans ceux de case.

Trois raisons principales sont évoquées pour cette pratique. La diminution de l'ombre pour l'augmentation du rendement des cultures associées, le rajeunissement de l'arbre afin d'accroître sa production de fruits et la lutte contre les parasites. Le bois issu de cette pratique contribue à la satisfaction des besoins énergétiques et de construction.

\section{Les techniques de régénération du parc à Vipalogo}

Après leur construction, les parcs agroforestiers traditionnels de Vipalogo, s'enrichissent progressivement en espèces ligneuses.

L'ensemble des agriculteurs participent à cet enrichissement qui s'effectue par la plantation ou la régénération naturelle assistée (RNA) ou par les deux modes à la fois. 3,1\% des personnes enquêtées plantent des arbres dans les champs, 71,9\% 
augmentent la densité du parc après sa construction par la RNA et enfin $25 \%$ pratiquent les deux techniques de régénération simultanément.

La RNA consiste à épargner les rejets, les repousses et à les protéger contre les animaux (Photos 4 et 5). Elle concerne aussi bien les champs de case que les champs de brousse. $97 \%$ des producteurs pratiquent cette technique pour la reconstitution du potentiel ligneux dans le parc. Cette pratique a été enseignée dans le terroir par les services forestiers. Elle concerne essentiellement les espèces locales.

Pour ce qui est de la plantation, 28\% des producteurs la pratiquent. Parmi eux, $89,3 \%$ plantent uniquement dans les champs de case et $10,7 \%$ dans les champs de case et de brousse. On distingue la plantation de type délimitation (autour des champs) pour marquer les limites de propriété, réalisée par $35,5 \%$ des personnes enquêtées et la plantation de type plein champ (arbres disséminés à l'intérieur des champs), effectuée par 51,6\%. Enfin, 12,9\% associent les deux types de plantation.

Le choix des producteurs pour les espèces à planter porte essentiellement sur des espèces exotiques dont Azadirachta indica représente environ $42 \%$ en nombre d'individus plantés. Les autres espèces ligneuses sont par ordre de préférence: Mangifera indica, Eucalyptus camaldulensis, Psidium guajava, Citrus aurantifolia (Christm.) Swingle, Gmelina arborea Roxb. et Moringa oleifera Lam. (Arbonnier, 2002).

Les modes d'acquisition des plants sont l'achat dans la pépinière villageoise dans $66,6 \%$ des cas, le don dans $16,7 \%$ et les prélèvements dans les bas-fonds pour 16,7\% des cas. Les espèces prélevées dans les basfonds sont généralement des jeunes pieds de manguiers ou de neem dont les graines ont trouvé des conditions favorables pour pousser.

Le mode de régénération du parc pratiqué est fonction du statut foncier de l'exploitant (Tableau 2). Il ressort de ce Tableau que le seul mode de régénération utilisé par ceux qui ont emprunté des terres est la RNA. Par contre, dans le cas des propriétaires terriens, les deux modes sont utilisés avec un fort pourcentage toutefois pour la RNA.

\section{Utilisation des produits du parc}

De nombreux besoins de la population sont satisfaits à partir des arbres du parc. Ce sont les besoins en bois (bois de feu, d'œuvre, de service), en médecine traditionnelle et en alimentation. Le tableau ci-après présente l'ensemble des espèces inventoriées et leurs différentes utilisations dans le terroir de Vipalogo (Tableau 3).

Dans le terroir de Vipalogo, plusieurs espèces ligneuses contribuent à satisfaire les besoins quotidiens en bois de chauffe. De ces espèces, dix interviennent pour $93.9 \%$ dans la couverture de ces besoins. Au niveau du bois de service (piliers ou poutres pour les hangars et la toiture des cases et greniers), une vingtaine d'espèces ligneuses sont couramment utilisées. $88,1 \%$ des besoins sont couverts par seulement dix espèces. Pour le bois d'œuvre, 26 espèces sont utilisées, et dont les dix principales couvrent $86,2 \%$ des besoins.

\section{Besoins en pharmacopée traditionnelle Pharmacopée humaine}

Plusieurs espèces du parc interviennent dans le traitement des maladies chez l'homme. Parmi ces espèces, dix contribuent pour $60,9 \%$ à la fréquence totale d'utilisation (Tableau 4)

Les feuilles, fruits, racines et écorces sont les principales parties de l'arbre utilisées, (Figure 2). L'écorce est la partie de l'arbre la plus utilisée avec $46 \%$, suivi des feuilles $40 \%$, les racines interviennent pour $12 \%$.

\section{Pharmacopée animale}

A Vipalogo, selon les enquêtés, vingt espèces ligneuses du parc sont utilisées pour traiter les maladies chez les animaux dont dix espèces contribuent pour plus de $75 \%$ à la satisfaction de ces besoins (Tableau 5). 
Comme dans le cas de la pharmacopée humaine, les écorces, les racines, les feuilles, les fruits, les tiges et les fleurs sont diversement utilisés (Figure 3). Pour la pharmacopée animale, ce sont les écorces qui interviennent le plus avec $66 \%$ des cas, suivi des feuilles $15 \%$. Les racines et les fruits ont la même représentativité avec $8 \%$ chacun.

\section{Besoins alimentaires}

Certaines essences ligneuses du parc agroforestier de Vipalogo interviennent dans l'alimentation des populations. En effet, les fruits sont consommés à l'état frais ou transformés et jouent un rôle d'aliments d'appoint (Vitellaria paradoxa, Parkia biglobosa, Lannea microcorpa...). Les fleurs et les feuilles, quant à elles, sont généralement utilisées comme condiments dans la préparation des sauces.

L'exploitation des produits non ligneux repose essentiellement sur la cueillette, activité qui consiste à collecter les feuilles, fleurs et fruits au moyen de certains outils tels la gaule, le bâton, la hache et la machette. Ce sont surtout les feuilles, les fruits, les fleurs et les amandes qui sont exploités et consommés à l'état frais ou sec.

\section{Lieux de récolte des produits}

Les produits ligneux et non ligneux sont récoltés dans les champs de case, les champs de brousse, les jachères et la brousse. Pour les producteurs rencontrés, la notion de jachère recouvre les champs dont eux mêmes ont vu la mise en culture suivie de l'abandon, les autres formations naturelles dont ils n'ont pas souvenance d'avoir vu quelqu'un $y$ cultiver sont considérées comme brousses.

La Figure 4 donne la représentativité des différentes unités dans la satisfaction des populations en produits forestiers ligneux et non ligneux.

Toutes les unités de végétation interviennent dans la satisfaction des besoins des populations et cela à des degrés divers en fonction du type de produit recherché (Figure 4).
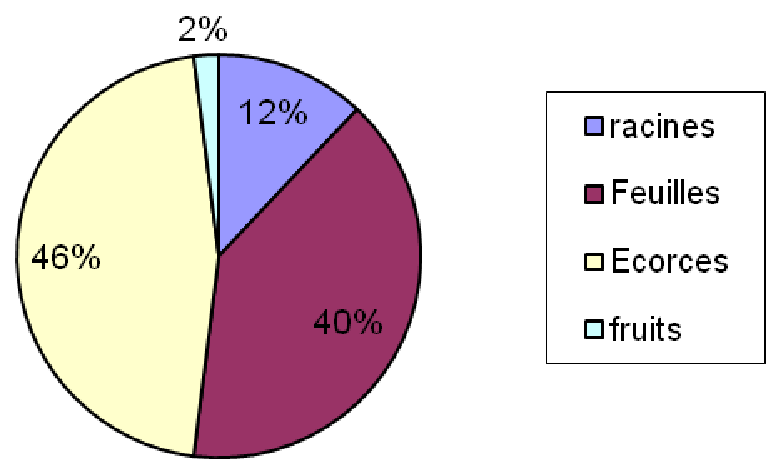

Figure 2: Spectre des organes des plantes utilisées dans la pharmacopée humaine à Vipalogo. 


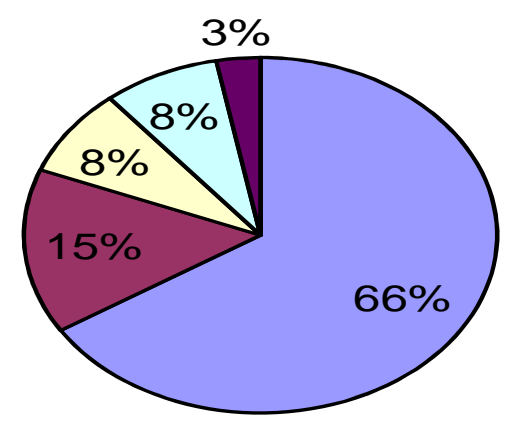

\section{$\square$ Ecorces \\ 口Feuilles \\ 口Racines \\ 口Fruits \\ a Fleurs}

Figure 3: Spectre des organes des plantes utilisées dans la pharmacopée animale à Vipalogo.

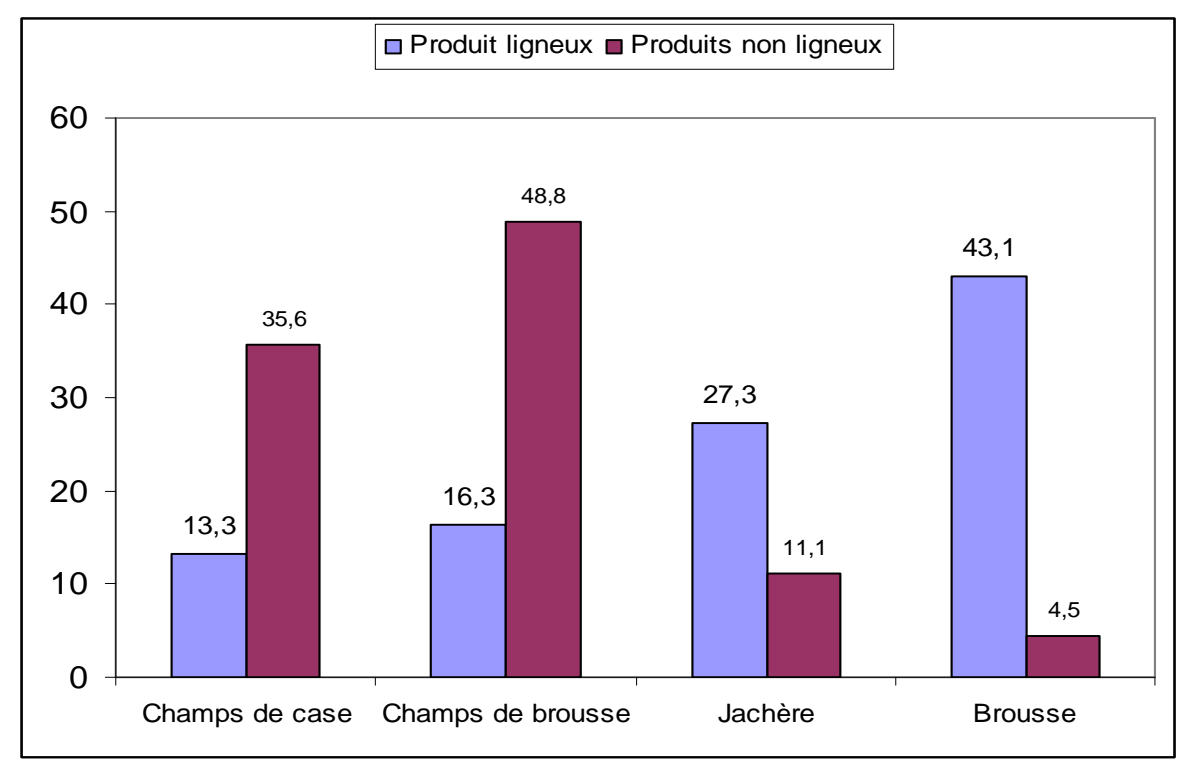

Figure 4: Contribution des différentes unités de végétation à la satisfaction des besoins des populations de Vipalogo. 


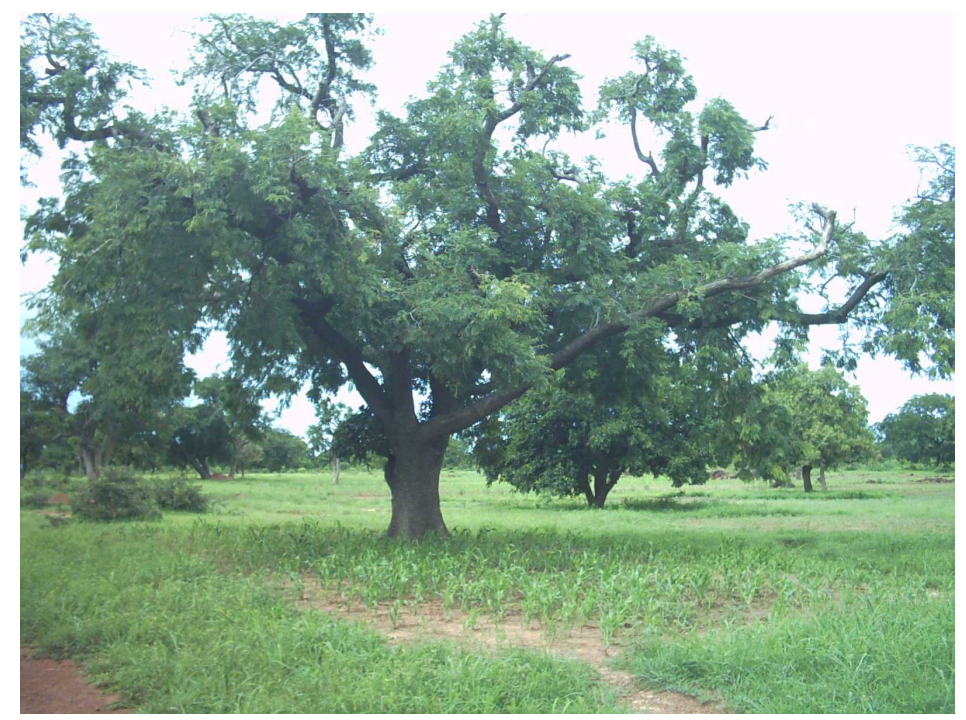

Photo 1: Aspect d'un néré partiellement émondé.

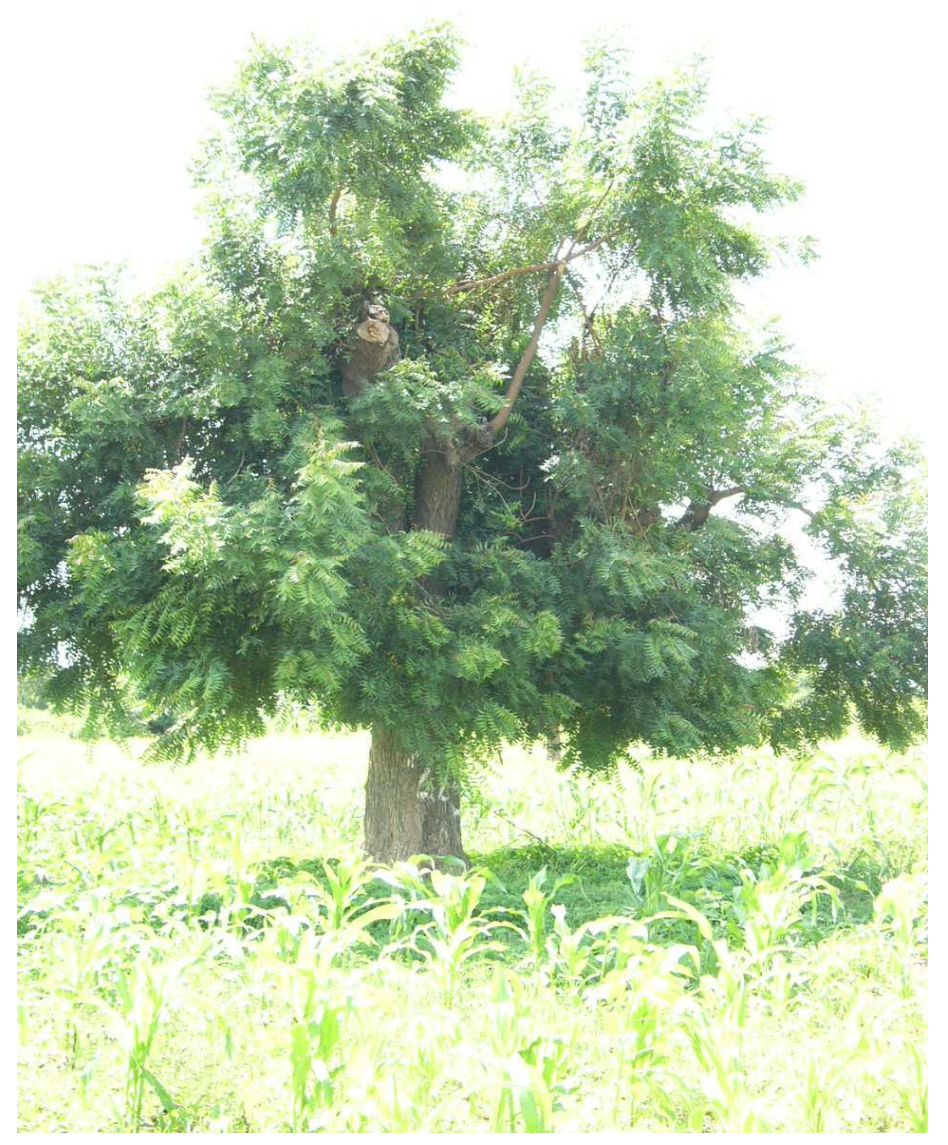

Photo 2: Repousse des branches d'un neem après étêtage à Vipalogo. 


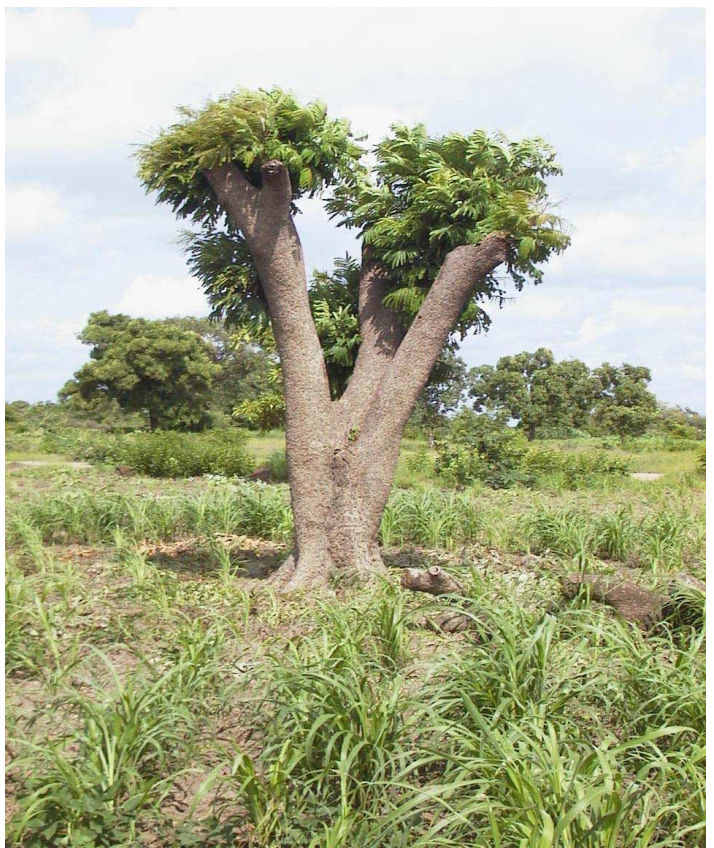

Photo 3 : Vieux pied de néré nouvellement étêté à Vipalogo.

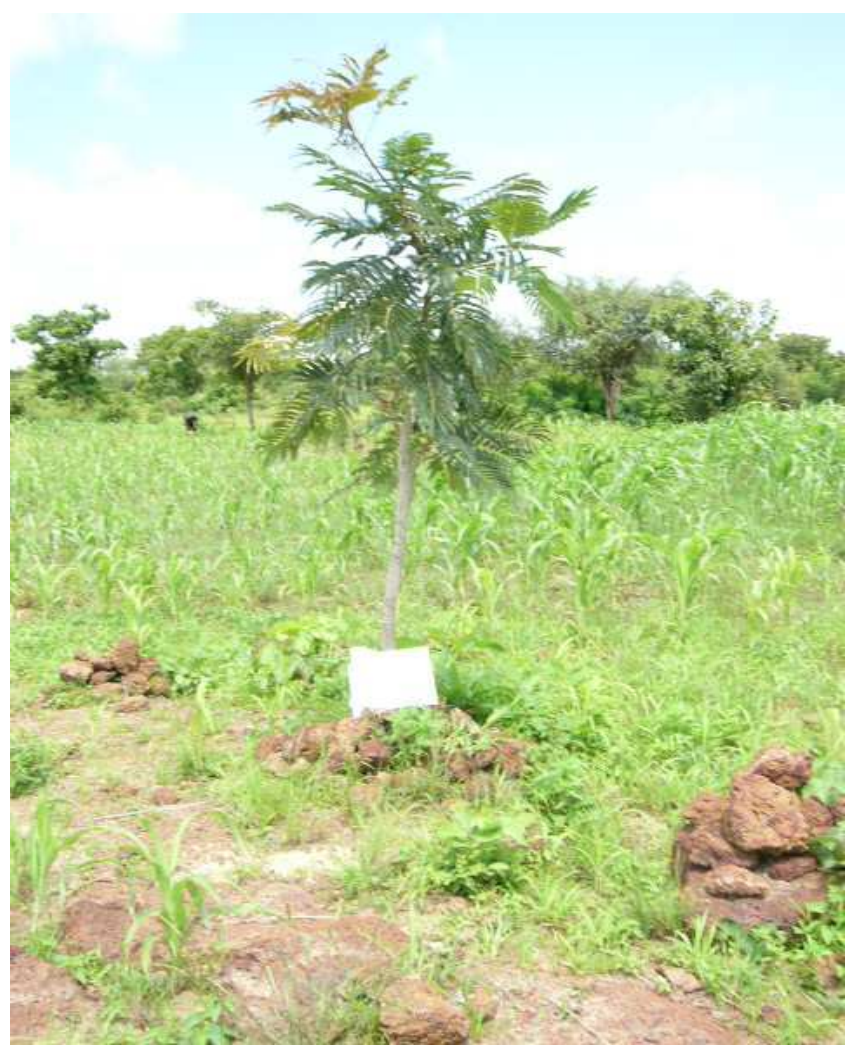

Photo 4: Jeune pied de Néré. 


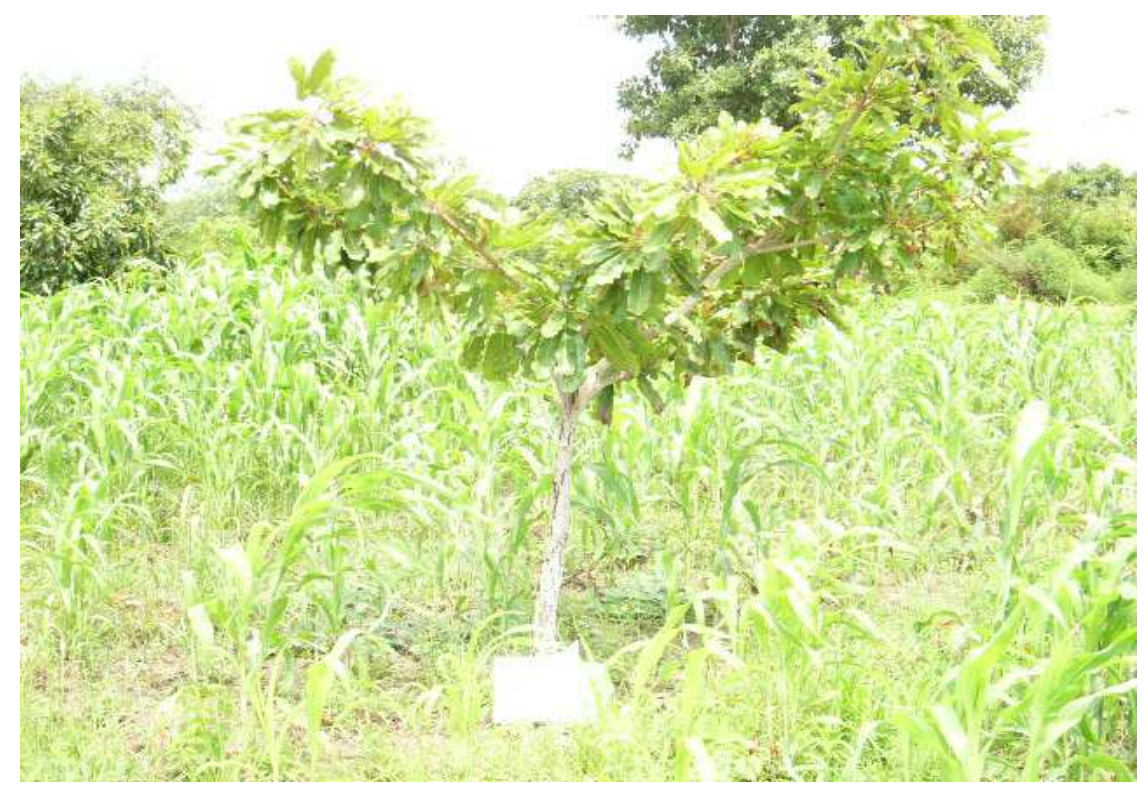

Photo 5 : Jeune pied de karité.

Tableau 1: Proportion des producteurs pratiquant l'émondage par type de champ à Vipalogo.

\begin{tabular}{lcc}
\hline Type de champs & $\begin{array}{c}\text { Nombre de } \\
\text { réponses positives }\end{array}$ & Proportion (en \%) \\
\hline Champ de case & 12 & 27,3 \\
Champ de brousse & 15 & 34,1 \\
Champ de case et de & 17 & 38,6 \\
brousse & & 100 \\
Total & 44 & \\
\hline
\end{tabular}

Tableau 2: Types de régénérations en fonction du statut foncier de l'exploitant.

\begin{tabular}{lccc}
\hline Statut du champ & \multicolumn{3}{c}{ Types de régénération (en \%) } \\
\cline { 2 - 4 } & Plantation & RNA & Plantation/RNA \\
\hline Propriétaire & 3,7 & 66,7 & 29,6 \\
Emprunt & 0 & 100 & 0 \\
\hline
\end{tabular}


G. YAMEOGO et al. / Int. J. Biol. Chem. Sci. 7(3): 1087-1105, 2013

Tableau 3: Espèces végétales des parcs agroforestiers de Vipalogo et les différentes utilisations.

\begin{tabular}{|c|c|c|c|c|c|c|c|c|c|c|}
\hline \multirow{3}{*}{ Espèces végétales } & \multicolumn{10}{|c|}{ Usages en \%) } \\
\hline & \multirow[t]{2}{*}{ Bois de feu } & \multirow{2}{*}{$\begin{array}{l}\text { Bois de } \\
\text { service }\end{array}$} & \multirow[t]{2}{*}{ Bois d'oeuvre } & \multicolumn{4}{|c|}{ Alimentation humaine } & \multirow{2}{*}{$\begin{array}{c}\text { Alimentatio } \\
\text { n bétail }\end{array}$} & \multirow{2}{*}{$\begin{array}{c}\text { Pharmacopée } \\
\text { humaine }\end{array}$} & \multirow{2}{*}{$\begin{array}{c}\text { Pharmacopée } \\
\text { vétérinaire }\end{array}$} \\
\hline & & & & $\mathbf{F e}$ & Fr & Fl & $\mathbf{G r}$ & & & \\
\hline Acacia dudgeoni Craib. ex Hall. & + & + & 1.2 & NU & NU & $\mathrm{NU}$ & $\mathrm{NU}$ & NU & 0.8 & NU \\
\hline Acacia nilotica (L.) Willd. ex Del. & + & + & 1.3 & NU & NU & $\mathrm{NU}$ & $\mathrm{NU}$ & ++ & 1.0 & 2.1 \\
\hline Acacia senegal (L.) Willd. & + & 1.1 & 18.8 & NU & NU & NU & NU & + & 0.8 & 2.3 \\
\hline Acacia seyal Del. & + & NU & 3.4 & NU & NU & $\mathrm{NU}$ & $\mathrm{NU}$ & + & 0.8 & 2.2 \\
\hline Acacia sieberiana DC. & 0.1 & + & 1.0 & NU & NU & $\mathrm{NU}$ & NU & + & 1.2 & + \\
\hline Afzelia africana Smith ex Pers. & 0.2 & + & 0.7 & + & NU & NU & NU & +++ & 0.9 & 2.1 \\
\hline Anacardium occidentale $\mathrm{L}$. & + & + & + & NU & ++ & NU & ++ & NU & + & NU \\
\hline Anogeissus leiocarpus (DC) Guill. et Perr. & 0.5 & 19.1 & 14.8 & NU & NU & $\mathrm{NU}$ & $\mathrm{NU}$ & + & 0.8 & 14.3 \\
\hline Azadirachta indica & 8.9 & 12.9 & 9.4 & NU & NU & $\mathrm{NU}$ & $\mathrm{NU}$ & NU & 4.5 & 2.2 \\
\hline Balanites aegyptiaca (L.) Del. & + & + & 6 & + & ++ & + & $\mathrm{NU}$ & +++ & 1.1 & + \\
\hline Bombax costatum Pellegr. et Vuillet & NU & NU & NU & +++ & +++ & +++ & + & + & 0.8 & + \\
\hline Borassus aethiopum (Mart.) Warburg. & NU & NU & NU & NU & + & $\mathrm{NU}$ & NU & NU & + & NU \\
\hline Boswelia dalzieliiHutch. & NU & NU & NU & NU & NU & NU & $\mathrm{NU}$ & NU & + & NU \\
\hline Burkea africana Hook. F. & 0.1 & 21.9 & 8.1 & + & NU & $\mathrm{NU}$ & $\mathrm{NU}$ & $\mathrm{NU}$ & 7 & 2.4 \\
\hline Calotropis procera (Ait.) Ait.f. & NU & NU & NU & NU & NU & NU & NU & NU & 1.2 & 1.1 \\
\hline Cassia siamea (Lam.) Irwin et Barneby & 0.5 & + & + & NU & NU & $\mathrm{NU}$ & $\mathrm{NU}$ & NU & 1.0 & NU \\
\hline Cassia sieberiana DC. & 3.2 & 2.8 & NU & NU & NU & $\mathrm{NU}$ & NU & NU & 5.6 & 2.2 \\
\hline Citrus aurentifolia (Christm.) Swingle & + & NU & NU & NU & ++ & $\mathrm{NU}$ & $\mathrm{NU}$ & NU & 2.3 & + \\
\hline Combretum glutinosum Perr. ex DC. & 12.7 & 4.5 & 4.1 & NU & NU & $\mathrm{NU}$ & $\mathrm{NU}$ & + & 0.8 & 1.2 \\
\hline
\end{tabular}


G. YAMEOGO et al. / Int. J. Biol. Chem. Sci. 7(3): 1087-1105, 2013

\begin{tabular}{|c|c|c|c|c|c|c|c|c|c|c|}
\hline Combretum nigricans Lepr. ex Guill. et Perr. & 3.8 & + & 1.2 & NU & NU & $\mathrm{NU}$ & $\mathrm{NU}$ & + & 0.8 & 2.3 \\
\hline Crataeva religiosa Forst. F. & $\mathrm{NU}$ & $\mathrm{NU}$ & + & ++ & + & $\mathrm{NU}$ & + & + & 1.7 & + \\
\hline Detarium microcarpum Guill. et Perr. & 16.5 & 1.2 & 0.6 & $\mathrm{NU}$ & +++ & $\mathrm{NU}$ & $\mathrm{NU}$ & + & 4.9 & 2.2 \\
\hline Dichrostachys cinerea (L.) Wight et Arn. & 0.2 & + & 0.6 & NU & + & $\mathrm{NU}$ & + & + & 1.0 & + \\
\hline Diospyros mespiliformis Hochst. & 8.9 & 8.4 & 12.8 & $\mathrm{NU}$ & +++ & NU & $\mathrm{NU}$ & $\mathrm{NU}$ & 0.8 & 4.8 \\
\hline Entada africana Guill. et Perr. & 0.5 & $\mathrm{NU}$ & $\mathrm{NU}$ & NU & NU & $\mathrm{NU}$ & $\mathrm{NU}$ & + & 4.2 & $\mathrm{Nu}$ \\
\hline Erythrina senegalensis DC. & $\mathrm{NU}$ & NU & + & $\mathrm{NU}$ & NU & NU & $\mathrm{NU}$ & + & 0.8 & + \\
\hline Eucalyptus camaldulensis & 3.2 & 5.1 & 2.7 & $\mathrm{NU}$ & NU & $\mathrm{NU}$ & $\mathrm{NU}$ & $\mathrm{NU}$ & 6.3 & NU \\
\hline Faidherbia albida (Del.) Chev. & + & $\mathrm{NU}$ & + & $\mathrm{NU}$ & NU & $\mathrm{NU}$ & $\mathrm{NU}$ & +++ & 0.8 & + \\
\hline Ficus ingens (Miq.) Miq. & + & 1.2 & 0.8 & $\mathrm{NU}$ & + & $\mathrm{Nu}$ & $\mathrm{NU}$ & + & + & + \\
\hline Ficus platyphylla Del. & 0.2 & 0.8 & + & $\mathrm{NU}$ & + & NU & $\mathrm{NU}$ & +++ & 0.8 & + \\
\hline Ficus sycomorus(Miq.) C. C. Berg. & 0.1 & 2.2 & + & + & + & NU & NU & +++ & 0.8 & + \\
\hline Gmelina arborea Roxb. & + & + & NU & NU & NU & NU & NU & $\mathrm{NU}$ & NU & NU \\
\hline Guiera senegalensis J.F. Gmel. & 15.2 & $\mathrm{NU}$ & + & $\mathrm{NU}$ & NU & $\mathrm{NU}$ & $\mathrm{NU}$ & $\mathrm{NU}$ & 4.2 & 2.4 \\
\hline Khaya senegalensis (Desr.) A. Juss. & 0.5 & 0.5 & 0.5 & $\mathrm{NU}$ & NU & NU & $\mathrm{NU}$ & +++ & 1.3 & 4.8 \\
\hline Lannea acida A. Rich. & + & 1.5 & 1.2 & NU & ++ & $\mathrm{NU}$ & NU & $\mathrm{NU}$ & 1.0 & + \\
\hline Lannea microcarpa & 0.5 & 1.6 & 0.3 & $\mathrm{NU}$ & +++ & NU & $\mathrm{NU}$ & + & 0.8 & + \\
\hline Lannea velutina A. Rich. & + & $\mathrm{NU}$ & 0.8 & NU & ++ & $\mathrm{NU}$ & NU & + & 0.8 & NU \\
\hline Mangifera indica & + & $\mathrm{NU}$ & NU & NU & +++ & NU & NU & + & 0.8 & + \\
\hline Parkia biglobosa & 3.8 & NU & + & + & +++ & + & +++ & + & 9.1 & 2.4 \\
\hline Piliostigma reticulatum (DC.) Hochst. & 17.7 & 1.2 & 0.8 & + & $\mathrm{NU}$ & $\mathrm{NU}$ & $\mathrm{Nu}$ & + & 1.4 & 4.8 \\
\hline Piliostigma thonningii (Schumach.) Milne-Redh. & 0.5 & 1.0 & 0.6 & NU & + & NU & NU & + & 0.8 & + \\
\hline Prosopis africana (Guill. et Perr.) Taub. & 0.1 & 8.4 & 3.4 & $\mathrm{NU}$ & $\mathrm{NU}$ & $\mathrm{NU}$ & $\mathrm{NU}$ & +++ & 1.0 & 1.2 \\
\hline Psidium guajava & + & $\mathrm{NU}$ & $\mathrm{NU}$ & $\mathrm{Nu}$ & ++ & $\mathrm{NU}$ & $\mathrm{NU}$ & $\mathrm{NU}$ & 2.8 & 7.1 \\
\hline
\end{tabular}


G. YAMEOGO et al. / Int. J. Biol. Chem. Sci. 7(3): 1087-1105, 2013

\begin{tabular}{|c|c|c|c|c|c|c|c|c|c|c|}
\hline Pterocarpus erinaceus Poir. & 0.5 & + & + & NU & NU & $\mathrm{NU}$ & $\mathrm{NU}$ & ++ & 0.8 & 1.0 \\
\hline Sclerocarya birrea (A. Rich.) Hochst. & 0.1 & + & 8.7 & NU & + & NU & + & + & 0.8 & + \\
\hline Securidaca longepedunculata Fres. & + & NU & NU & NU & NU & NU & $\mathrm{NU}$ & NU & 1.7 & + \\
\hline Sterculia setigera Del. & $\mathrm{NU}$ & NU & $\mathrm{NU}$ & NU & NU & NU & $\mathrm{NU}$ & + & 1.0 & $\mathrm{NU}$ \\
\hline Stereospermum kunthianum Cham. & $\mathrm{NU}$ & + & 0.5 & NU & NU & NU & NU & +++ & 1.0 & 1.8 \\
\hline Strychnos spinosa Lam. & + & + & + & + & + & NU & $\mathrm{NU}$ & + & + & + \\
\hline Swartzia madagascariensis Desv. & + & 2.8 & 0.6 & NU & NU & NU & NU & NU & 8 & 19 \\
\hline Tamarindus indica $\mathrm{L}$. & + & + & + & +++ & +++ & +++ & $\mathrm{NU}$ & +++ & 1.9 & + \\
\hline Terminalia avicennioüdes Guill. et Perr. & + & + & NU & NU & NU & $\mathrm{NU}$ & NU & NU & 0.8 & $\mathrm{NU}$ \\
\hline Thevetia neriifolia Juss. & $\mathrm{NU}$ & NU & NU & NU & NU & NU & NU & NU & NU & $\mathrm{NU}$ \\
\hline Trichilia roka (Forssk.) Chiov. & + & $\mathrm{Nu}$ & 0.4 & NU & NU & NU & $\mathrm{NU}$ & NU & 0.8 & $\mathrm{NU}$ \\
\hline Vitellaria paradoxa & 1.5 & 1.8 & 0.7 & NU & +++ & NU & +++ & $\mathrm{NU}$ & 11.9 & 11.9 \\
\hline Ziziphus mauritiana Lam. & + & + & + & NU & ++ & + & NU & +++ & 1.0 & + \\
\hline
\end{tabular}

$\mathrm{Fe}=$ Feuille $; \mathrm{Fl}=$ Fleur $; \mathrm{Gr}=$ Graine $; \mathrm{Fr}=$ Fruit $; \mathrm{NU}=$ Non utilisé $; \ll+»=$ Utilisation occasionnelle $;++=$ utilisation fréquente $;+++=$ Beaucoup utilisé

$\square$ Représente les dix principales espèces suivant les types d'utilisation. 
G. YAMEOGO et al. / Int. J. Biol. Chem. Sci. 7(3): 1087-1105, 2013

Tableau 4: Principales espèces végétales et maladies essentielles traitées en pharmacopée humaine.

\begin{tabular}{|c|c|c|c|c|c|}
\hline Espèces & $\begin{array}{l}\text { Représen- } \\
\text { tativité (\%) }\end{array}$ & Feuilles & Ecorce & Racines & Fleur/Fruit \\
\hline Vitellaria paradoxa & 11.9 & Trouble nerveux & Anti- venimeux & - & - \\
\hline Parkia biglobosa & 9.1 & Bronchite, Zona,Fièvre & Hemoroïde & Stérilité Maladies vénériennes & Gengivite \\
\hline Burkea africana & 7 & Hémoroïde,Fissures annales & - & - & - \\
\hline $\begin{array}{l}\text { Eucalyptus } \\
\text { camaldulensis }\end{array}$ & 6.3 & Etat febrile, Bronchite & - & - & - \\
\hline Cassia sieberiana & 5.6 & Abcès & Troubles hépato-biliaires & $\begin{array}{c}\text { Maux de ventre, Troubles } \\
\text { hépatiques }\end{array}$ & - \\
\hline Azadirachta indica & 4.5 & Paludisme, Oedèmes Rhumatismes & - & - & - \\
\hline Detarium microcarpum & 4.9 & Malnutrition des enfants & Epilepsie & - & - \\
\hline Entada africana & 4.2 & & Fortifiant & Toux & \\
\hline Guiera senegalensis & 4.2 & Diarrhée, Rhume,Toux & - & - & Toux, Rétention urinaire \\
\hline Psidium guajava & 2.8 & Diarrhée, Rafermie les dents & $\begin{array}{c}\text { Leucorrhée Hémorragie } \\
\text { utérienne }\end{array}$ & - & - \\
\hline
\end{tabular}


Tableau 5: Principales espèces végétales et maladies essentielles traitées en pharmacopée animale.

\begin{tabular}{|c|c|c|c|c|c|}
\hline Espèces & $\begin{array}{c}\text { Représenta- } \\
\text { tivité }(\%)\end{array}$ & Feuilles & Ecorces & Racines & Fleur / Fruit \\
\hline Swartzia madagascarensis & 19 & Laxatives Fièvre & Laxatives Ictères & - & Diarrhée Otite \\
\hline Vitellaria paradoxa & 11.9 & Ictère Constipation Fièvre & Diarrhée Toux & Ascite Stérilité & Diarrhée Antivenimeux \\
\hline Anogeissus leiocarpus & 14.3 & Ictère Diarrhées & Vermifuge Otite Toux & Hernie Toux & $\begin{array}{c}\text { Anorexie (Ane) } \\
\text { Ténifuges (Chevaux) }\end{array}$ \\
\hline Psidium guajava & 7.1 & $\begin{array}{c}\text { Coliques Astringents } \\
\text { Diarrhées }\end{array}$ & - & Antiseptiques Vaginites & Dysenterie Ictère \\
\hline Diospyros mespiliformis & 4.8 & - & $\begin{array}{l}\text { Antifongique } \\
\text { Antihémorragique }\end{array}$ & & Pneumonie \\
\hline Khaya senegalensis & 4.8 & - & $\begin{array}{c}\text { Ulcère Pneumonie Otite } \\
\text { Stérilité }\end{array}$ & Vermifuges Purgatives & Gastrite Fièvre \\
\hline Piliostigma reticulatum & 4.8 & Toux Bronchite Fièvre & $\begin{array}{c}\text { Bronchite Colique } \\
\text { Désinfectant }\end{array}$ & Vermifuges Purgatives & $\begin{array}{c}\text { Conjonctivite Plaie et } \\
\text { blessure }\end{array}$ \\
\hline Parkia biglobosa & 2.4 & Vermifuges Abcès & $\begin{array}{c}\text { Ulcère Pneumonie Otite } \\
\text { Stérilité }\end{array}$ & Vermifuges & Anorexie Ictère \\
\hline Burkea africana & 2.4 & Fièvre & & Douleurs abdominales & Diarrhée \\
\hline Guiera senegalensis & 2.4 & - & Coliques & Bronchite Diarrhée & - \\
\hline
\end{tabular}




\section{DISCUSSION}

Le terroir de Vipalogo appartient au système agro-sylvo-pastoral Nord soudanien. Dans ce terroir, cinq principales pratiques sylvicoles sont couramment appliquées dans le parc: la coupe partielle des arbres, la coupe complète, l'émondage, la régénération naturelle assistée et la plantation.

La coupe partielle s'effectue aussi bien dans les champs de case que dans ceux de brousse. C'est un mode d'exploitation des espèces fruitières (Parkia biglobosa, Lannea microcarpa, Bombax costatum, Tamarindus indica...) dont les branches sont très développées ou très hautes. Les branches coupées sont celles portant le plus de feuilles ou de fruits, en vue de satisfaire les besoins du moment.

L'émondage vise le rajeunissement des branches de l'arbre afin d'accroître sa production fruitière. Il contribue également à la réduction de l'effet de l'ombrage sur les cultures et également à lutter contre les parasites (Bayala, 2002) dont les effets néfastes sur le développement des arbres ont été notés par plusieurs auteurs dont Sallé et al. (1998) et Boussim et al. (1995). L'émondage concerne surtout les espèces agroforestières traditionnelles et fruitières telles que Parkia biglobosa, Vitellaria paradoxa, Bombax costatum, Tamarindus indica. Parmi les espèces exotiques concernées par cette pratique, figure Azadirachta indica. Si pour la plupart des espèces locales la recherche des fruits conditionne l'émondage, la pratique sur Azadirachta indica a pour principal objectif l'utilisation du feuillage comme mulch dans les parties encroûtées des champs. C'est une pratique très connue dans la région comme l'ont constaté Bationo et al. (2004).

La plantation et la Régénération Naturelle Assistée (RNA) sont les modes d'enrichissement du parc arboré à Vipalogo. Cependant, seuls les propriétaires terriens peuvent planter. L'arbre apparait comme une source de droit foncier. Planter est une manière de matérialiser le droit de propriété. Pour Le Roy et al. (1996) parmi les pressions qui s'exercent sur ceux qui ont prêté des terres, il y a la restriction concernant la plantation. Dans le terroir de Vipalogo, les mêmes constats prévalent. Toutefois, même pour les propriétaires terriens, la pratique n'est pas très répandue et s'effectue surtout dans les champs de case. Les espèces plantées à Vipalogo sont des espèces exotiques soit fruitières (Mangifera indica, Psidium guajava, Citrus aurantifolia), soit à production rapide de bois (Eucalyptus camaldulensis, Gmelina arborea, Azadirachta indica...), pour palier à la forte pression sur les ligneux. Elles sont généralement plantées en alignement ou en bordure des champs (limite des propriétés). Ce type de plantation se justifie par la petitesse des superficies et permettent à certains de matérialiser les limites de propriété. De plus, le souci de réduire l'effet de l'ombrage sur les cultures a été évoqué.

La Régénération Naturelle Assistée concerne surtout les espèces locales. Planter des arbres n'est pas dans la culture des populations du terroir. En effet, pour reprendre les mots d'un vieux du terroir: «les espèces locales, on les connaît. Elles ne sont pas plantées, on les laisse pousser naturellement». La RNA est beaucoup plus pratiquée dans les champs de brousse. L'objectif visé par l'utilisation des différents modes de régénération est de satisfaire les nombreux besoins des populations aussi bien en produits forestiers ligneux que non ligneux. Si les produits non ligneux interviennent le plus souvent pour l'alimentation, les produits ligneux quant à eux, peuvent être utilisés comme bois de feu, bois d'œuvre ou de service (Yaméogo, 2009). Il faut noter que tout arbre existant au champ, qu'il provienne de la plantation ou de la RNA, appartient au propriétaire terrien avec la possibilité de jouir des fruits ou du bois par l'emprunteur sauf dans le cas de Parkia biglobosa qui reste une propriété exclusive du propriétaire terrien. 
En ce qui concerne le bois de feu, les principales espèces ligneuses utilisées à Vipalogo sont des espèces qui ne produisent pas de fruits comestibles à quelques exceptions près. Pour les espèces du parc produisant des fruits comestibles et que l'on retrouve citées comme fournissant du bois de feu, il s'agit des branches issues de l'élagage, de l'émondage, ou de l'étêtage lors de la récolte des fruits, ou pendant les coupes de rajeunissement. La production de bois de feu par ces espèces constitue un résultat secondaire. C'est le cas de Parkia biglobosa dont le bois utilisé pour la cuisine provient des branches coupées lors de la récolte des fruits. Le karité n'est pas cité par les populations comme étant utilisé en bois de feu. Toutefois, dans la pratique, on constate que son bois sert à la cuisson des repas soit par utilisation directe ou par transformation en charbon de bois. Le fait de ne l'avoir pas cité au cours de l'enquête tient au fait que sa coupe est formellement interdite par les services forestiers et tout contrevenant s'il est pris s'expose à des sanctions car l'espèce est intégralement protégée.

Pour les populations rencontrées, l'utilisation des arbustes notamment les Combretaceae comme bois de feu s'explique par le fait que leur bois coupé vert, sèche rapidement et que de plus, ce sont des essences qui régénèrent vite. $\mathrm{La}$ bonne régénération des espèces ligneuses en zone tropicale a été notée par Ribot (2001).

Le parc participe pour beaucoup à la satisfaction des besoins des exploitations en bois de service et en bois d'œuvre. Le besoin à satisfaire peut conditionner le choix des espèces.

C'est ainsi qu'en ce qui concerne le bois d'œuvre, pour la confection des équipements agricoles, (manches de daba, de haches, machettes), des objets d'art, les espèces à bois dur sont privilégiées. On y retrouve des espèces telles que Diospyros mespiliformis, Anogeissus leiocarpus,
Prosopis africana, Balanites aegyptiaca. Ici également, le karité n'est pas cité mais on sait également que son bois sert à la fabrication entre autre de mortier et de pilon.

Pour ce qui concerne le bois de service, notamment dans le domaine de la construction (habitat, hangars, grenier...), la résistance aux termites est privilégiée quand bien même la résistance physique joue également un rôle dans les choix. C'est ainsi qu'à ce niveau, on rencontre des espèces comme Anogeissus leiocarpus, Balanites aegyptiaca, Combretum micranthum, Diospyros mespiliformis, Azadirachta indica, Eucalyptus camaldulensis, Prosopis africana. Les espèces exotiques Azadirachta indica, Eucalyptus camaldulensis, plantées surtout dans les champs de case contribuent pour beaucoup à la satisfaction des besoins en bois de service, d'où l'importance des coupes complètes constatées sur ces espèces.

Parmi les raisons qui justifient le choix des espèces à conserver dans les champs, il y a les besoins en produits alimentaires et pharmaceutiques. En effet, le milieu naturel regorge d'importantes ressources que l'homme exploite pour se soigner, pour accompagner les produits de culture ou, pour palier aux insuffisances de provisions en période de soudure (Yélémou et al., 2007 ; Thiombiano et al., 2012). Le parc agroforestier du terroir de Vipalogo joue ce rôle. Pour les lieux de récolte, on constate que la grande partie des produits forestiers non ligneux est récoltée dans les champs de case et les champs de brousse. Plus de $84,4 \%$ des produits forestiers non ligneux sont récoltés dans les champs dont $51,2 \%$ dans les champs de case. Il y a donc comme une volonté de la part des producteurs de créer les conditions de proximité afin de trouver dans le champ une grande partie des produits nécessaires à leur alimentation.

La contribution des ressources ligneuses est importante également en ce qui concerne les produits de la pharmacopée, que 
ce soit pour la santé humaine ou animale. Les parties de l'arbre les plus utilisées sont les écorces et les racines. Ces prélèvements peuvent être préjudiciables à la survie des arbres s'ils sont mal réalisés ou réalisés de manière intense.

$\mathrm{Au}$ regard des différents besoins à satisfaire, la plupart des espèces du parc sont à usages multiples avec une importante place pour les espèces exotiques comme Azadirachta indica et Eucalyptus camaldulensis. En ce qui concerne les espèces locales, les plus utilisées à Vipalogo sont : Detarium microcarpum, Afzelia africana, Parkia biglobosa Vitellaria paradoxa, Lannea microcarpa, Bombax costatum.

\section{Conclusion}

La gestion du parc agroforestier obéit à une certaine logique paysanne tout comme sa création. Le mode de gestion utilisé vise à répondre à de nombreux objectifs : palier à la baisse des rendements agricoles consécutifs aux effets négatifs de l'ombrage sur les cultures, répondre aux besoins alimentaires, de pharmacopée traditionnelle et également de bois d'énergie.

Les différentes pratiques paysannes ont un effet sur la dynamique de la végétation ligneuse, donc sur la densité des parcs agroforestiers où ils trouvent la satisfaction de nombreux besoins. En outre, la majeure partie des espèces locales utilisées et/ou surexploitées sont en voie de disparition ou ont une régénération difficile. C'est pourquoi, il apparaît nécessaire de travailler à promouvoir des modes de gestion efficaces afin d'insuffler un meilleur dynamisme au parc agroforestier.

\section{REFERENCES}

Arbonnier M. 2002. Arbres, Arbustes et Lianes des Zones Sèches d'Afrique de l'Ouest ( $2^{\text {ème }}$ édition). CIRAD MNHN ; $573 \mathrm{p}$.
Bationo BA, Yélémou B, Ouédraogo SJ. 2004. Le neem (Azadirachta indica A. Juss.), une espèce exotique adoptée par les paysans du centre ouest du Burkina Faso. Bois et Forêts des Tropiques, 284(4): 5-10.

Bayala J. 2002. The crown purning as a management tool to enhance the productivity of parkland in West Africa. PhD Thesis, School of Agriculture and Forest Sciences, University of Wales, Bangor, UK, p. 209.

Boussim JI, Guinko S, Sallé G, Rayal-Roques A. 1995. Le parasitisme de Thapinantus sur le karité au Burkina Faso. Nuisibles-PestsPragas, 3(1): 164-172.

ENGREF. 2001. Gestion des ligneux dans la région de MAFA-KILDA, Nord Cameroun. Voyage d'étude de la Formation Forestière Rurale et Tropicale, ENGREF-IRAD/PRASAC, Montpellier, p. 38.

Fontes J, Guinko S. 1995. Carte de la végétation et de l'occupation du sol du Burkina Faso. Notice explicative, Ministère de la coopération française, projet Campus, Toulouse, p. 68.

Gautier D, Tebaya O, Nounga E, Mana J. 2001. Femmes du Nord Cameroun et ressources arborées: usages, perceptions de l'évolution et perspectives d'actions dans les 5 villages de références du PRASAC, IRAD/PRASAC/CIRAD, Maroua, 110 p.

Le Roy E, Karsenty A, Bertrand A. 1996. La Sécurité Foncière en Afrique. Pour une Gestion Viable des Ressources Renouvelables. Edition Karthala: Paris.

MEDEV/ DGEP. 2005. Situation économique et financière du Burkina Faso en 2004 et tendances pour 2005-2008, MEDEV/ DGEP.

Millogo-Rasolodimby J. 2001. L'homme, le climat et les ressources alimentaires en période de crise de subsistance au cours du $20^{\mathrm{è}}$ siècle au Burkina Faso. Thèse de 
Doctorat d'Etat, Université de Ouagadougou, Ouagadougou, p. 248.

PANA (Programme d'action national d'adaptation à la variabilité et aux changements climatiques). 2006. Rapport provisoire. Ministère de l'Environnement et du Cadre de Vie, Ouagadougou.

Ribot J. 2001. Historique de la Gestion Forestière en Afrique de l'Ouest. Comment la 'Science' Exclut les Paysans. IIED: Angleterre, London; p. 17.

Sallé G, Tuquet C, Rayal-Roques A. 1998. Biologie des phanérogames parasites. Soc. Biol., 192: 9-36.

Thiombiano DNE, Lamien N, Dibong DD, Boussim IJ, Belem B. 2012. Le rôle des espèces ligneuses dans la gestion de la soudure alimentaire au Burkina Faso. Sécheresse, 23: 86- 93.

Thiombiano A. 2005. Les Combretaceae du Burkina Faso: taxonomie, écologie, dynamique et régénération des espèces. Thèse d'Etat, Université de Ouagadougou, p. 290.

Traoré S, Thiombiano L, Millogo JR, \& Guinko S. 2007. Carbon and nitrogen enhancement in Cambisols and Vertisols by Acacia spp. in eastern Burkina Faso: Relation to soil respiration and microbial biomass. Applied Soil Ecology, 35: 660669.

Yaméogo G, Yélémou B, Traoré D. 2005. Pratique et perception paysannes dans la création de parc agroforestier dans le terroir de Vipalogo (Burkina Faso).
Biotechnol. Agron. Soc. Environ., 4: 241248.

Yaméogo G. 2009 : Les ressources ligneuses et leur gestion dans le terroir de Vipalogo, province du Kadiogo, Burkina Faso. Mémoire de thèse Unique de Doctorat, Université de Cocody-Abidjan, Côte D'Ivoire, p. 224.

Yaro E. 2000. Potentialités et possibilités de gestion participative des formations naturelles protégées : cas de la zone agrosylvo-pastorale de Sablogo (Province du Boulgou et du Koulpélogo au Burkina Faso). Mémoire IDR, Université Polytechnique de Bobo-Dioulasso, BoboDioulasso, p. 75.

Yélémou B. 2010. Biologie et écologie des espèces du genre Piliostigma et leur contribution à la dynamique de la matière organique des sols en zone SahéloSoudanienne au Burkina Faso. Mémoire de Thèse Unique, Université de Ouagadougou, Ouagadougou, p. 150.

Yélémou B, Bationo B, Yaméogo G, Millogo-Rasolodimby J. 2007. Gestion traditionnelle et usage de Piliostigma reticulatum (D.C.) Hochst., dans le Plateau central du Burkina Faso. Bois et Forêt des Tropiques, 291(1): 55-65. 